\title{
PSGL-1-mediated activation of EphB4 increases the proangiogenic potential of endothelial progenitor cells
}

\author{
Philippe Foubert, ${ }^{1,2}$ Jean-Sébastien Silvestre, ${ }^{2}$ Boussad Souttou, ${ }^{1,2}$ Véronique Barateau, ${ }^{2}$ \\ Coralie Martin, ${ }^{3}$ Téni G. Ebrahimian, ${ }^{2}$ Carole Leré-Déan,, ${ }^{1,2}$ Jean Olivier Contreres, ${ }^{1,2}$ Eric Sulpice, ${ }^{1,2}$ \\ Bernard I. Levy, ${ }^{2}$ Jean Plouët,, ${ }^{1,2}$ Gérard Tobelem,1,2 and Sophie Le Ricousse-Roussanne ${ }^{1,2}$ \\ 1 Institut des Vaisseaux et du Sang, Paris, France. '2Cardiovascular Research Center, INSERM Lariboisière U689, \\ Paris VII, Paris, France. ${ }^{3}$ Museum National d'Histoire Naturelle, USM 307, Paris, France.
}

\begin{abstract}
Endothelial progenitor cell (EPC) transplantation has beneficial effects for therapeutic neovascularization; however, only a small proportion of injected cells home to the lesion and incorporate into the neocapillaries. Consequently, this type of cell therapy requires substantial improvement to be of clinical value. Erythropoietin-producing human hepatocellular carcinoma $(\mathrm{Eph})$ receptors and their ephrin ligands are key regulators of vascular development. We postulated that activation of the EphB4/ephrin-B2 system may enhance EPC proangiogenic potential. In this report, we demonstrate in a nude mouse model of hind limb ischemia that EphB4 activation with an ephrin-B2-Fc chimeric protein increases the angiogenic potential of human EPCs. This effect was abolished by EphB4 siRNA, confirming that it is mediated by EphB4. EphB4 activation enhanced P selectin glycoprotein ligand-1 (PSGL-1) expression and EPC adhesion. Inhibition of PSGL-1 by siRNA reversed the proangiogenic and adhesive effects of EphB4 activation. Moreover, neutralizing antibodies to $E$ selectin and $P$ selectin blocked ephrin-B2-Fc-stimulated EPC adhesion properties. Thus, activation of EphB4 enhances EPC proangiogenic capacity through induction of PSGL-1 expression and adhesion to E selectin and P selectin. Therefore, activation of EphB4 is an innovative and potentially valuable therapeutic strategy for improving the recruitment of EPCs to sites of neovascularization and thereby the efficiency of cell-based proangiogenic therapy.
\end{abstract}

\section{Introduction}

Peripheral vascular disease is a major health problem in Western countries. In the late stages of this disease, progression of tissue hypoperfusion results in ischemic ulceration and gangrene, leading to amputation in more than a third of patients. Thus, prevention and treatment are 2 important and urgent goals for the management of tissue ischemia. Endothelial progenitor cell (EPC) transplantation improves neovascularization of ischemic hind limbs and ischemic hearts owing to their capacity to integrate new blood vessels and/or to secrete proangiogenic factors (1-4). Recent clinical trials illustrate the potential for the use of BM-derived or circulating blood-derived progenitor cells to improve blood flow and function of ischemic tissues (5). Although cell-based therapeutic strategies have several attractive features for tissue regeneration, their efficacy is limited by the pathological microenvironment and the fact that few of the intravenously injected cells accumulate at sites of tissue damage (6). Therefore, development of novel proangiogenic strategies that improve revascularization of ischemic tissues are needed (7-9).

Erythropoietin-producing human hepatocellular carcinoma (Eph) receptors and ephrins are membrane proteins that are classified into 2 broad subclasses, A and B, according to their structural homologies and binding specificities. Eph receptors belong

Nonstandard abbreviations used: BMMC, BM mononuclear cell; DAB, 3,3'-diaminobenzidine; EPC, endothelial progenitor cell; Eph, erythropoietin-producing human hepatocellular carcinoma; PIGF-1, placental growth factor-1; PSGL-1, P selectin glycoprotein ligand-1; SDF-1 $\alpha$, stromal cell-derived factor $1 \alpha$; TBST, Tris-buffered saline containing $0.1 \%$ Tween- 20 .

Conflict of interest: The authors have declared that no conflict of interest exists. Citation for this article: J. Clin. Invest. 117:1527-1537 (2007). doi:10.1172/JCI28338. to the largest family of receptor tyrosine kinases and they autophosphorylate upon binding to their cognate ephrin ligands. Ephrin-B ligands are transmembrane proteins that preferentially bind to receptors of the EphB subclass. Originally identified as neuronal guidance molecules, Eph and ephrin are also expressed by a number of nonneural cells, including ECs, hematopoietic cells, and tumor cells (10). During the early stages of vascular development, EphB4 is specifically expressed in venous endothelium whereas ephrin-B2 is expressed in arterial endothelium (11). Studies in mouse embryos showed that these 2 proteins are essential for embryonic heart development and angiogenesis. Indeed, targeted null mutations in EphB4 and ephrin-B2 genes caused embryonic lethality with defects in angiogenic remodeling of the peripheral vasculature and myocardial trabeculation (11-13). Interaction between Eph receptors and ephrins requires cell-cell contacts because both molecules are anchored to the plasma membrane. The resulting signals propagate bidirectionally into both the Eph receptor-expressing cells (forward signaling) and the ephrin-expressing cells (reverse signaling). The interaction between EphB4 and ephrin-B2 is thought to play an important role not only in cell-cell and cell-ECM interactions but also in cell migration, adhesion, and proliferation (14).

As EPCs are involved in postnatal vasculogenesis (1-4), we sought to determine whether activation of EphB4 forward signaling modulates their angiogenic properties in a mouse model of hind limb ischemia. Our results show that activation of EPCs with an ephrin-B2-Fc chimeric protein enhanced, in an EphB4dependent manner, therapeutic neovascularization of hind limb ischemia through upregulation of $\mathrm{P}$ selectin glycoprotein ligand-1 (PSGL-1) and binding to E selectin and P selectin. 
A
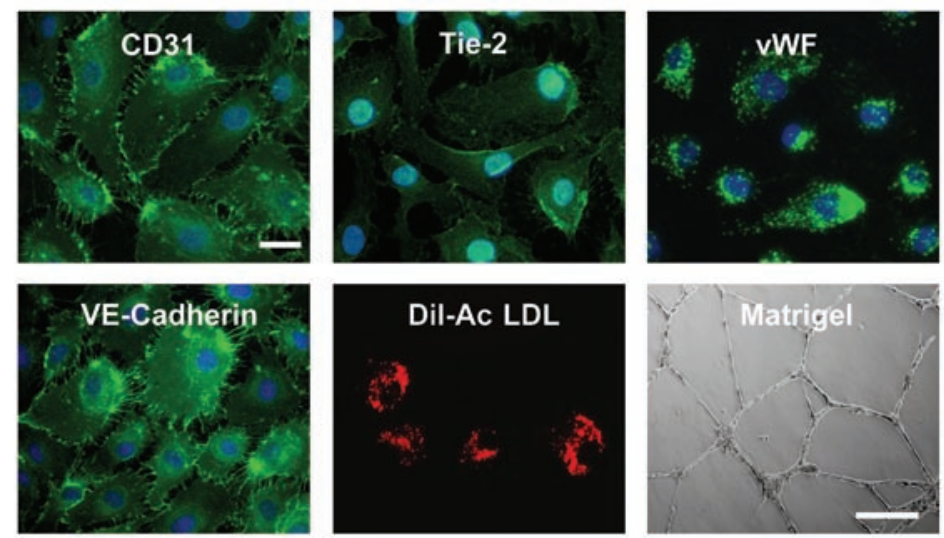

B
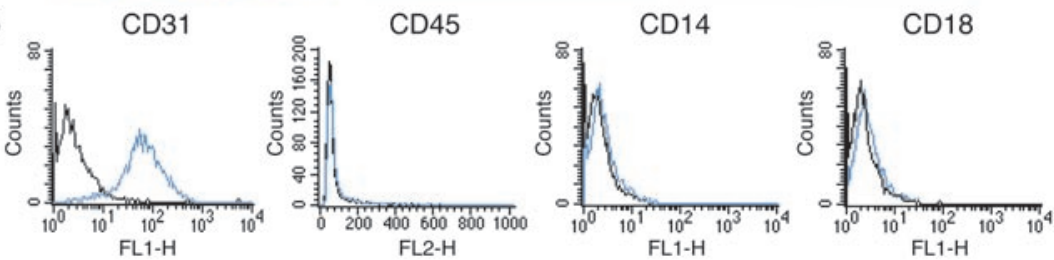

\section{Figure 1}

Phenotypic and functional characterization of EPCs. (A) EPCs were fixed in $90 \%$ cold acetone and incubated with the appropriate primary antibody, then with a FITC-coupled secondary antibody. EPCs were characterized according to the presence or absence of endothelial-specific markers including CD31, Tie-2, vWF, vascular endothelial-cadherin (VE-cadherin), and uptake of Dil-acetylated LDL (Dil-Ac LDL) (scale bar: $10 \mu \mathrm{m}$ ) and by their capacity to induce tube formation on Matrigel (scale bar: $100 \mu \mathrm{m}$ ). (B) Flow cytometric analysis of surface antigens on EPCs. EPCs were positive for CD31 but not for monocytic markers CD45, CD14, and CD18 (blue histogram). Isotypic control is represented by the black histogram.

\section{Results}

\section{Phenotypic and functional characterization of EPCs derived from umbilical cord blood}

We isolated and differentiated EPCs from human cord blood in the presence of VEGF-A. These cells displayed an endothelial phenotype: they expressed CD31, Tie-2, vascular endothelial-cadherin, and vWF (Figure 1A). They also incorporated Dil-acetylated LDL and formed capillary-like structures on Matrigel (Figure 1A). FACS analysis revealed that at least $98 \%$ of the total population expressed endothelial markers, indicating that our EPC cultures were homogenous (for details, see ref. 15). These cells were positive for CD31 but negative for the monocytic markers CD45, CD14, and CD18 (Figure 1B).

\section{Expression of EphB4 in EPCs and its activation by an ephrin-B2-Fc chimera}

In order to determine whether activation of the EphB4 receptor in EPCs enhances their proangiogenic potential, we first assessed the expression of ephrin-B2 and EphB4 in these cells. EPCs expressed EphB4 and its ligand ephrin-B2 at both mRNA and protein levels (ref. 15 and Supplemental Figure 1; supplemental material available online with this article; doi:10.1172/ JCI28338DS1). We then analyzed the capacity of an ephrin-B2-Fc chimeric protein to activate EphB4 downstream signaling. Ephrin-B2-Fc increased EphB4 phosphorylation, as detected by Western blotting with an anti-phosphotyrosine antibody (Figure 2A). As EphB4 activation is followed by receptor-ligand clustering and its internalization (16), we performed double-positive staining for both EphB4 and phosphotyrosine on cultured cells. The data showed that the intensity of the phosphotyrosine signal was substantially higher in the ephrin-B2-Fc-treated cells than in control cells. In the ephrin-B2-Fc-treated cells, the EphB4 and the phosphotyrosine signals colocalized mostly as clusters at the cell membrane and as endocytosed particles (Figure 2B). Thus, EPCs derived from human umbilical cord blood expressed functional EphB4 tyrosine kinase receptors that can be activated by an ephrin-B2-Fc chimera.

As it is well established that EphB4 and ephrin-B2 dictate arterial-venous identity (11), we sought to determine whether EPCs can assume arterial or venous phenotypes following EphB4 activation. EPCs were treated with ephrin-B2-Fc for 6 hours or 24 hours and then analyzed for EphB4 and ephrin-B2 expression at both mRNA and protein levels (Supplemental Figure 2). RT-PCR and immunofluorescence analyses indicated that ephrin-B2-Fc stimulation did not induce EPC specification toward arterial or venous phenotype.

\section{EphB4 activation in EPCs enhanced their neovascularization potential in hind limb ischemia}

We next determined whether activation of EphB4 signaling enhances the therapeutic effect of EPCs. For this purpose, EPCs were stimulated with either ephrin-B2-Fc, EphB4-Fc, or CD6-Fc fusion proteins and were intravenously injected into mice that underwent femoral artery ligature. Since EPCs do not express the CD6 ligand, ALCAM (activated leukocyte cell adhesion molecule) or CD166, the CD6-Fc fusion protein was used as a negative control.

Localization of the injected EPCs in ischemic muscle. We used doublefluorescence labeling to detect injected EPCs in sections of the gastrocnemius muscle. Human EPCs were stained with an antihuman CD31 antibody (red labeling) whereas mouse vasculature was stained with an anti-mouse CD31 antibody (green labeling). Our results indicated that transplanted EPCs incorporated into the mouse vasculature (Figure 3 ). In addition, the number of incorporated EPCs, as detected with Dil labeling, was higher following ephrin-B2-Fc stimulation (Supplemental Figure 3).

Angiographic score. Transplantation of EPCs raised the ischemic/ nonischemic angiographic score by $47.5 \%$ over that of control animals injected with PBS $(P<0.01)$ (Figure 4). Vessel density following infusion of ephrin-B2-Fc-stimulated EPCs was $34.2 \%$ and $98 \%$ higher than that of mice injected with untreated EPCs $(P<0.001)$ or PBS $(P<0.001)$, respectively (Figure $4 \mathrm{~A})$. EphB4-Fc pretreat- 
A

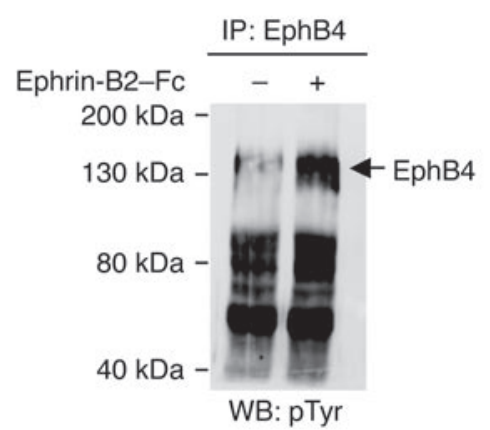

WB: EphB4
B
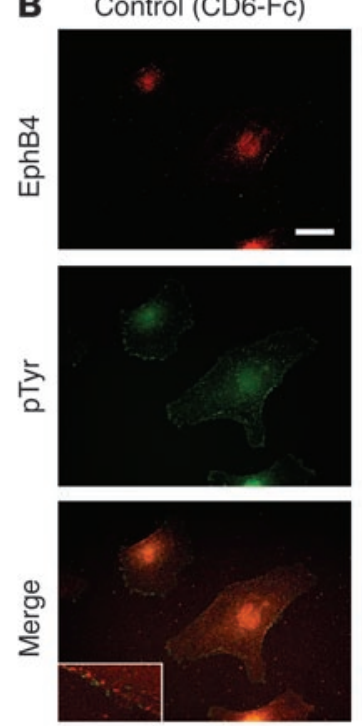

+ Ephrin-B2-Fc
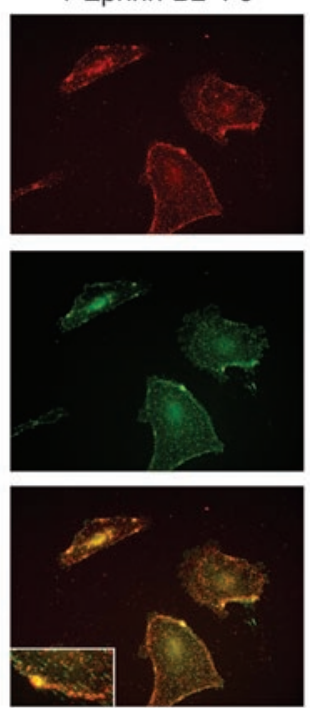

Figure 2

EphB4 activation by ephrin-B2-Fc. (A) EPCs were stimulated with $3 \mu \mathrm{g} / \mathrm{ml}$ ephrin-B2-Fc for 30 minutes at $37^{\circ} \mathrm{C}$. Cell lysates were prepared, subjected to immunoprecipitation with an antihuman EphB4 antibody, and resolved by SDS-PAGE, and proteins were transferred to nitrocellulose membranes as described in Methods. Membranes were then blotted with the $4 \mathrm{G} 10$ antiphosphotyrosine antibody. To check for equal protein loading, membranes were stripped and reprobed with an anti-EphB4 antibody. pTyr, phosphotyrosine; WB, Western blot. (B) To confirm EphB4 activation, ephrin-B2-Fc-stimulated EPCs were subjected to double immunostaining with EphB4 and anti-phosphotyrosine antibodies. Note the membrane colocalization of the EphB4 and the 4G10 signals corresponding to clusters (yellow color) in ephrin-B2-stimulated cells. Inset: magnification showing clustering and internalization of the phosphorylated EphB4 when cells were stimulated with ephrin-B2-Fc (right) but not with control CD6-Fc (left). Scale bar: $10 \mu \mathrm{m}$.

ment to stimulate ephrin-B2 pathways did not affect EPC-related effects. Similarly, CD6-Fc pretreatment had no effect.

Capillary density. When mice were transplanted with nonstimulated EPCs, capillary density was increased by $54.7 \%$ compared with that of mice injected with PBS (Figure 4B). Capillary density was higher by $160.4 \%$ and $96.3 \%$ in mice transplanted with ephrin-B2-Fcstimulated EPCs compared with that of PBS- and EPC-injected mice, respectively $(P<0.001)$. Cells stimulated with either EphB4-Fc or CD6-Fc had the same effects as nonstimulated EPCs.

Laser Doppler perfusion imaging. Intravenous delivery of EPCs increased the ischemic/nonischemic foot blood flow ratio by $41 \%$ with reference to control animals receiving PBS $(P<0.01)$ (Figure 4C). Injection of EPCs stimulated by ephrin-B2-Fc resulted in hind limb blood flow recovery $93 \%$ greater than that in PBSinjected animals $(P<0.001)$. It also improved foot perfusion by $41 \%$ as compared with injection of nonstimulated EPCs $(P<0.01)$. No such improvement was detected when cells were stimulated with either EphB4-Fc or CD6-Fc. These results demonstrate that activation of EphB4 by ephrin-B2-Fc strongly enhanced the therapeutic potential of EPCs in nude mice with hind limb ischemia.

\section{RNA interference targeting EphB4 inbibited the proangiogenic activity induced by the ephrin-B2-Fc fusion protein}

Ephrin-B2 can bind to and signal through EphB2, EphB3, and EphB4. However, EphB4 is generally considered to be specific for ephrin-B2. To determine whether EphB4 activation drives the ephrin-B2-Fc-enhanced EPC proangiogenic effects, we targeted upregulates the secretion of the angiogenic factors stromal cell-derived factor $1 \alpha$ (SDF-1 $\alpha)$, VEGF-A, and placental growth factor-1 (PlGF-1).

Ephrin-B2-Fc stimulation of EPCs did not enhance migration toward $V E G F-A$. To determine whether activation of EphB4 by ephrin$\mathrm{B} 2-\mathrm{Fc}$ influences EPC migration, we used a modified Boyden chamber system. In this migration assay, EPCs were treated with the various fusion proteins and then allowed to migrate toward VEGF-A. None of the recombinant proteins (ephrin-B2-Fc, EphB4-Fc, and $\mathrm{CD} 6-\mathrm{Fc}$ ) influenced EPC migration in response to VEGF-A (Supplemental Figure 4).

Ephrin-B2-Fc stimulation of EPCs did not induce secretion of angiogenic factors. Growth factor levels were measured by ELISA in EPC-conditioned media. No release of SDF-1 $\alpha$ or VEGF-A was detected in conditioned media of activated EPCs (data not shown). In contrast, PlGF-1 was detected in conditioned media of nonactivated EPCs, but its concentration was unaffected by ephrin-B2-Fc or EphB4-Fc pretreatment (Supplemental Figure 5). These observations indicate that EphB4-related effects were not mediated by upregulation of these proangiogenic factors.

EphB4 activation in EPCs enhanced adhesiveness to a HUVEC monolayer. To analyze the effect of EphB4-activation on EPC adhesion capabil$i t y$, we used an IL-1 $\beta$-activated monolayer of HUVEC. In this model, IL- $1 \beta$ increases the expression of various adhesion molecules, including the selectins, ICAM-1, and VCAM-1. EPCs were stimulated with the various chimeric proteins (ephrin-B2-Fc, EphB4-Fc, and $\mathrm{CD} 6-\mathrm{Fc}$ ) and then added to IL-1 $\beta$-activated HUVEC. The number of adhering cells was $32.5 \%$ higher for ephrin-B2-Fc-treat- 


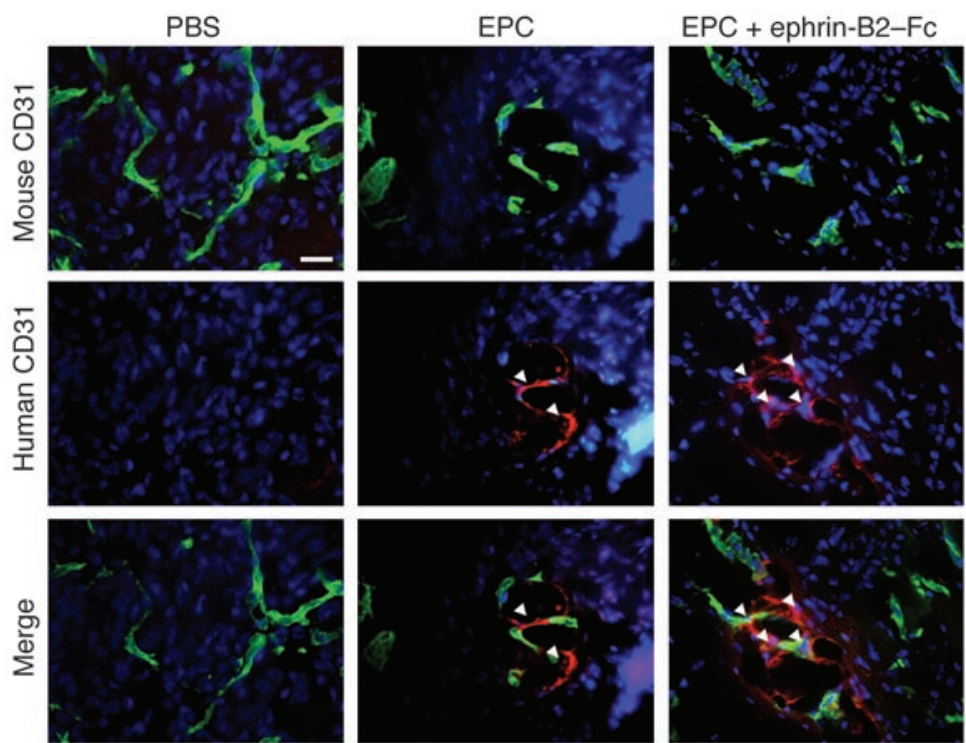

Figure 3

Transplanted EPCs home to the ischemic muscle. Representative photomicrographs of incorporated EPCs identified by double-fluorescence labeling in ischemic muscles. Transplanted human EPCs were stained using a biotinylated anti-human CD31 antibody (red fluorescence) in histological sections retrieved from ischemic muscles 4 days after injection. Mouse vasculature was identified by CD31 staining (green fluorescence) in the same tissue sections. Nuclei were stained with DAPI (blue labeling). Arrows indicate labeled EPCs. Scale bar: $50 \mu \mathrm{m}$.

ed than for untreated EPCs $(P<0.05)$ (Figure 6). Treatment with EphB4-Fc or CD6-Fc did not affect EPC adhesion. More interestingly, the ephrin-B2-Fc-related effects were completely abolished by transfection of the EPCs with an EphB4 siRNA, indicating that EPC adhesion was mediated by EphB4 activation (Figure 6).

EPhB4 activation enhanced EPC adhesion through PSGL-1. PSGL-1 (CD162) is a glycoprotein expressed at the surface of various hematopoietic cells $(20,21)$. It is a ligand for $\mathrm{E}$ selectin and $\mathrm{P}$ selectin (CD62-E, CD62-P), both of which are expressed at the surface of ECs, especially in the ischemic endothelium (20-22). Whereas none of the various treatments influenced expression of the adhesion molecules CD49b, CD49d, CD49e, and CD51/CD61 (data not shown), expression of PSGL-1 was 15.4-fold higher in ephrin-B2-Fc-treated than in untreated EPCs $(P<0.01)$ (Figure $7, \mathrm{~A}$ and $\mathrm{B})$. Transfection with an EphB4 siRNA abolished the ephrin-B2-Fc-induced expression of PSGL-1 (Figure 7, A and B), indicating that EphB4 activation controls PSGL-1 expression in EPCs. In contrast, stimulation with EphB4-Fc or CD6-Fc did not affect PSGL-1 expression.

We next sought to define the role of PSGL-1 in EPC adhesion following activation of the EphB4 pathway by ephrin-B2-Fc. For this purpose, we used siRNA directed against the PSGL-1 transcript. Immunofluorescence analysis indicated that PSGL-1 siRNA greatly impaired PSGL-1 expression in ephrin-B2-Fc-stimulated cells (Figure 7C). Subsequently, ephrin-B2-Fc-induced EPC adhesion was blocked by transfection with PSGL-1 siRNA (Figure 7D). More interestingly, inhibition of PSGL-1 also reversed the beneficial effect of ephrin-B2-Fc pretreatment on the proangiogenic capacity of EPCs in the ischemic hind limb model (Table 2). Taken together, these findings show that activation of the EphB4 pathway upregulated PSGL-1, leading to enhanced adhesion of EPCs to activated endothelium and to an increased proangiogenic potential of EPCs.

\section{Role of $E$ selectin and $P$ selectin/PSGL-1 interaction in EPC adbesion}

As PSGL-1 is a ligand for E selectin and P selectin, we assessed the expression of these selectins in ischemic and nonischemic muscles. E selectin and P selectin mRNA and protein were upregulated in ischemic muscles 6 hours after the onset of ischemia but not in nonischemic muscles at the same time interval and returned to their baseline level after 24 hours (Figure 8, A and B). We also analyzed PSGL-1 expression in EPCs 6 hours and 24 hours after ephrin-B2-Fc stimulation. PSGL-1 protein content was increased after 6 hours of stimulation and tended to decrease after 24 hours of exposure to ephrin-B2-Fc (Figure 8C). We next sought to determine whether upregulation of PSGL-1 by ephrin-B2-Fc increased EPC adhesion to immobilized $\mathrm{E}$ or $\mathrm{P}$ selectin-Fc proteins. EphB4-Fc or CD6-Fc treatment did not affect EPC adhesion to either selectins (Figure 9A). Conversely, the numbers of ephrin-B2-Fc-treated EPCs adhering to E selectin-Fc- or P selectin-Fc-coated plates were $45 \%(P<0.01)$ and $25 \%(P<0.05)$, respectively, higher than the numbers for untreated EPCs. In addition, ephrin-B2-Fc-induced EPC adhesion to IL-1 $\beta$-stimulated HUVEC was reduced after cotreatment with E selectin-blocking antibody and, to a lesser extent, with $\mathrm{P}$ selectin-blocking antibody (Figure 9B). Therefore, activation of EphB4 with ephrin-B2-Fc increased EPC adhesion through upregulation of PSGL-1 expression and interaction with E selectin and $\mathrm{P}$ selectin. Taken together, these results also suggest that the selectin/PSGL-1 interaction is required for the initial events of the neovascularization process.

\section{Discussion}

In the present study, we report that activation of EphB4 with an ephrin-B2-Fc chimeric protein enhanced the proangiogenic capacity of EPCs in a mouse hind limb ischemia model through induction of PSGL-1 expression and adhesion to E selectin and P selectin. Our results showed that ephrin-B2-Fc-treated EPCs were more potent than untreated or EphB4-Fc-treated EPCs for therapeutic revascularization. Supporting this finding, Hayashi et al. demonstrated that administration of ephrin-B2-Fc alone stimulated postnatal neovascularization in the murine cornea (23). It is likely that ephrin-B2-Fc would also stimulate EphB4-positive EPCs in this latter model.

Other EphB receptors that can interact with ephrin-B2 are also expressed on vascular ECs (12). In our experimental conditions, EPCs expressed not only EphB4 but also EphB2 and EphB3 (data not shown). Although ephrin-B2 binds EphB4 with high specificity, it can also bind EphB2 and EphB3, but with a far lower affinity (24). Using an siRNA targeting EphB4, we were able to completely abolish the ephrin-B2-Fc-induced proangiogenic activity. This demonstrated that ephrin-B2-Fc exerted its effects mainly through activation of the EphB4 receptor.

To elucidate the cellular and molecular mechanisms associated with the activation of EPCs, we assessed whether EphB4 activation influenced EPC migration in modified Boyden chamber assays. Our results showed that stimulation of EPCs with ephrin-B2-Fc did not influence migration toward VEGF-A in our assay conditions.

Recent experiments suggest that in addition to the physical contribution of EPCs to newly formed vessels, the enhanced secretion of proangiogenic factors may be a supportive mechanism for the 
A

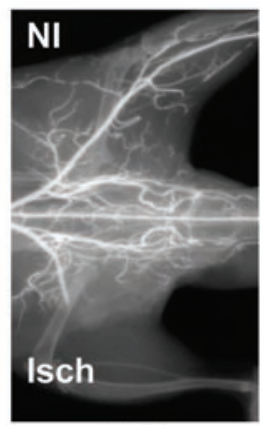

PBS

B
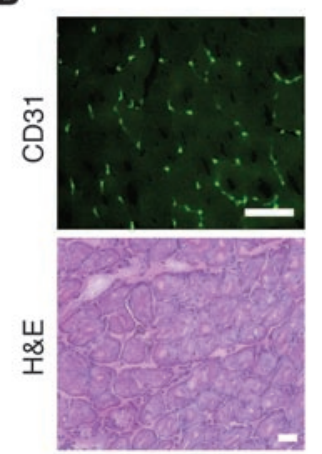

PBS

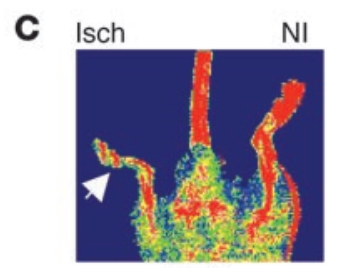

PBS

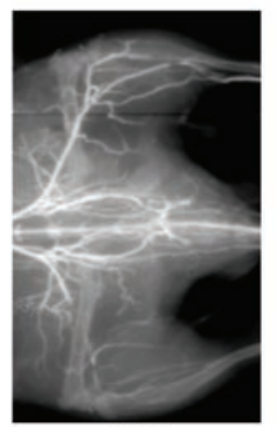

EPC
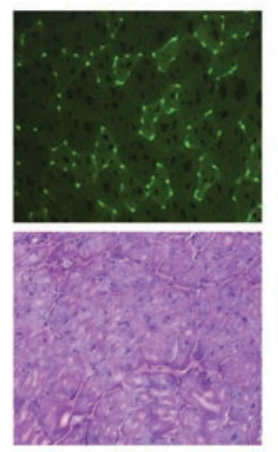

EPC

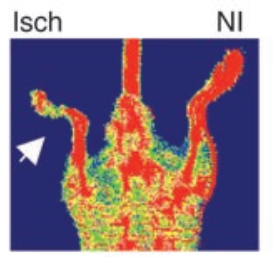

EPC

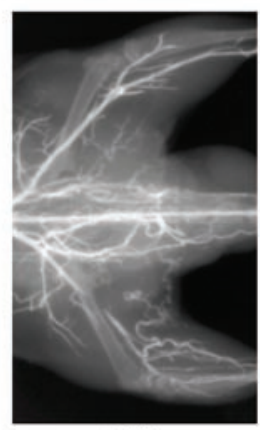

$\mathrm{EPC}+$

ephrin-B2-Fc
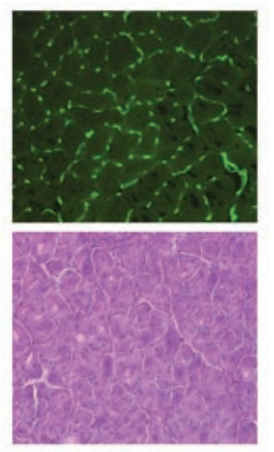

$E P C+$

ephrin-B2-Fc

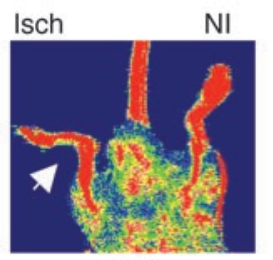

$E P C+$

ephrin-B2-Fc
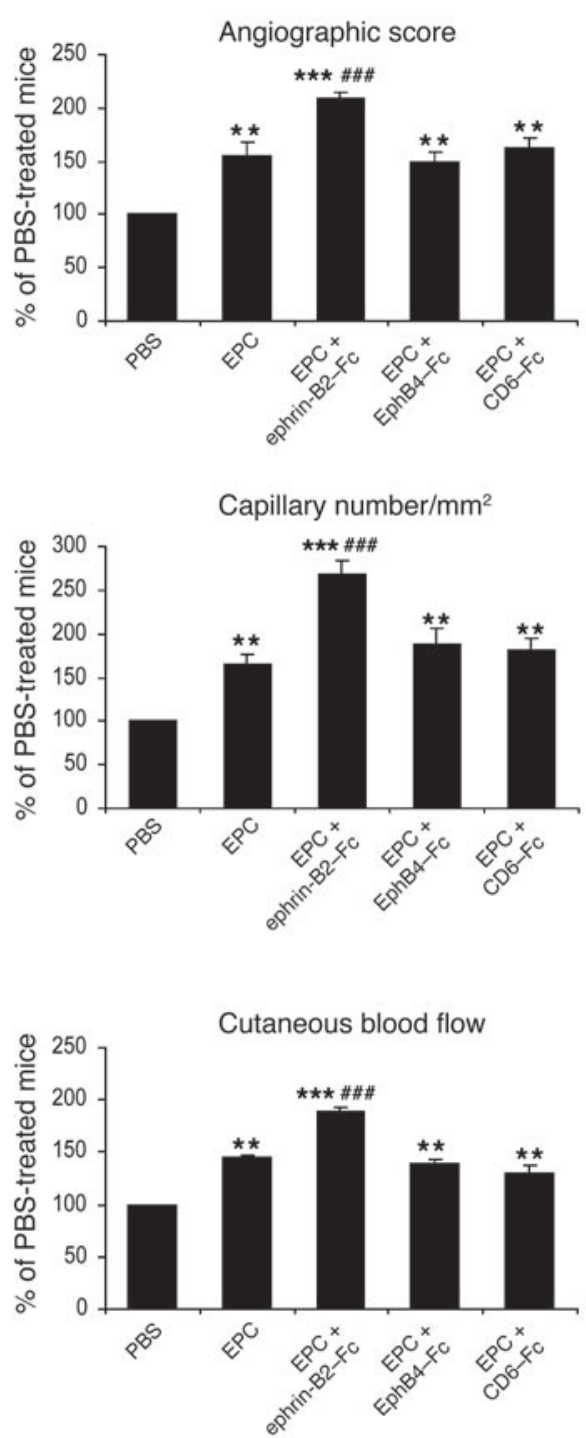

\section{Figure 4}

Ephrin-B2-Fc increases EPC proangiogenic potential in hind limb ischemia. (A) Representative photomicrographs and quantitative analysis of microangiography. (B) H\&E staining and capillary density. CD31-positive capillaries appear in green. Scale bar: $100 \mu \mathrm{m}$. (C) Foot perfusion in mice injected with PBS, nonstimulated EPCs (EPCs), and stimulated EPCs (EPCs + ephrin-B2-Fc, EPC + EphB4-Fc, or EPC + CD6-Fc). Values are expressed as means \pm SEM; $n=10$ per group. ${ }^{* *} P<0.01,{ }^{* * *} P<0.001$ versus PBS-injected mice; ${ }^{\# \#} P<0.01$, \#\#\# $P<0.001$ versus nonstimulated EPC-injected mice. NI, nonischemic; Isch, ischemic.

improvement of blood vessel formation (25). In our experiments, pretreatment with ephrin-B2-Fc fusion protein did not increase the secretion of SDF-1 $\alpha$, VEGF-A, or PlGF-1 by EPCs, suggesting that ephrin-B2-Fc-related effects were not mediated by paracrine activities of these factors. However, we cannot rule out the possibility that other angiogenic factors may be involved. During the homing process, EPCs may adhere to the endothelium at the ischemic site. We demonstrated that adhesion of EPCs to the endothelium may be further facilitated by EphB4 activation following ephrin-B2-Fc treatment. Modulation of integrin receptor expression may determine adhesiveness and thereby influence homing and arrest of EPCs at foci of ischemia or vascular injury (26). For example, the proan- giogenic effect of statin therapy could be explained by the increase in the number of circulating EPCs and an upregulation of integrin receptor subunits $C D 49 e, \beta 1, C D 51$, and $\beta 5$, resulting in enhanced EPC adhesion (27). In our model, expression of the adhesion molecules CD49b, CD49d, CD49e, and CD51/CD61 was not affected by activation of EphB4 with ephrin-B2-Fc. We report evidence that this effect was mediated by functional interaction between PSGL-1 and E and P selectin: (a) ephrin-B2-Fc induced expression of PSGL-1; (b) siRNA targeting PSGL-1 and antibodies neutralizing E selectin and P selectin impaired EPC adhesion to HUVEC monolayer; (c) PSGL-1 ligands E selectin and P selectin were induced at the site of ischemia; and, more importantly, (d) siRNA targeting PSGL-1 completely abol- 

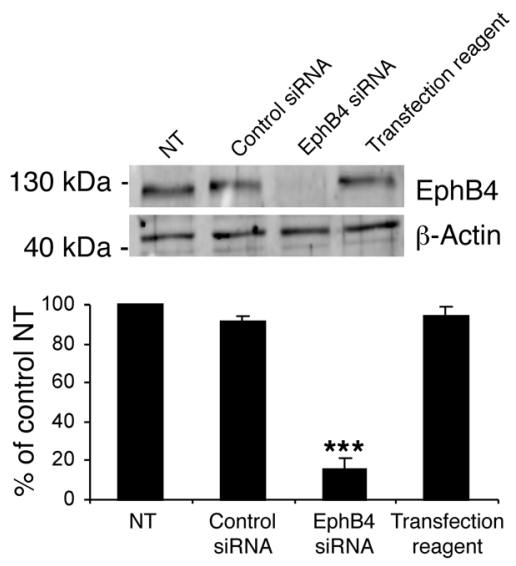

\section{Figure 5}

EphB4 knockdown in EPCs. Western blot quantification of EphB4 protein demonstrating the efficiency of EphB4 siRNA in transfected cells. The membrane was reprobed for $\beta$-actin to check for equal loading. NT, nontransfected cells; control siRNA, luciferase siRNA; transfection reagent, Dharmafect2. $n=3 .{ }^{* \star} P<0.001$ versus nontransfected cells.

ished the proangiogenic activity of ephrin-B2-Fc-stimulated EPCs in mouse hind limb ischemia. Using a tumor model system, Vajkoczy et al. demonstrated that selectins and their ligand PSGL-1 mediated the initial adhesion of EPCs to the tumor endothelium (28). In these experiments, pretreatment of mice with an antibody cocktail directed against E selectin and P selectin as well as pretreatment of EPCs with an antibody directed against PSGL-1 reduced the initial arrest of these cells at the tumor endothelium and their subsequent incorporation into the tumor microvasculature. Furthermore, human adult hematopoietic CD $34^{+}$stem cells express PSGL-1 and their homing was dramatically reduced (approximately 90\%) in selectin-deficient mice (29). P selectin/PSGL-1 interaction is also involved in the homing of T cell precursors from the BM to the thymus (30). These various observations implicate the selectin/PSGL-1 interaction as being important in the homing of EPCs. In our model, E selectin and P selectin were upregulated in ischemic areas, and PSGL-1 expression was enhanced in ephrin-B2-Fc-stimulated EPCs; it is therefore tempting to conclude that EphB4-driven expression of PSGL-1 increased EPC adhesion to activated endothelium and their arrest at foci of neovascularization (see the proposed model in Figure 10).

The cellular mechanisms leading to EphB4-induced upregulation of PSGL-1 expression remain to be defined. Stimulation of microvascular ECs with ephrin-B2-Fc results in the activation of Akt/ PI3K and ERK1/2 pathways (31), which may subsequently con- trol the EphB4-driven expression of PSGL-1 seen in our model. In addition, synthesis of bioactive PSGL-1 requires glycosylation and tyrosine sulfation for selectin recognition (32). These events are catalyzed by a series of dynamically regulated enzymes, including C2GnT-1 (core- $2 \beta 1,6-N$-acetylglucosaminyltransferase) and FucT-VII (fucosyltransferase). Therefore, it is possible that ephrinB2-Fc-increased PSGL-1 levels on EPCs results from enhanced transcription of C2GnT-1 or FucT-VII. However, we cannot exclude the possibility that other yet-undefined pathways can be involved.

Cell therapy for the treatment of ischemic diseases is currently a subject of intense investigation. However, various risk factors, including diabetes, hypercholesterolemia, and aging may limit its efficacy (5). Consequently, native neovascularization of the ischemic hind limb was remarkably delayed in old versus young animals (33). The number and the migratory activity of circulating EPCs have both been reported to be reduced in patients with risk factors for ischemic cardiovascular diseases, such as genetic predisposition or smoking (34). EPCs from patients with diabetes mellitus type 2 have less proliferative potential, impaired homing capacity, and lower ability for the formation of capillary tubes in vitro than those from healthy individuals (35). In type 1 diabetes, BM mononuclear cells (BMMCs) failed to differentiate into EPCs and to participate in vascular-like structure formation (36). Here, we demonstrate that ephrin-B2-Fc treatment enhanced the efficiency of EPC-based therapy. A number of clinical trials aimed at promoting neovascularization in ischemic myocardium and skeletal muscle have now been published (5). These trials were performed with transplantation of autologous progenitor cells: BMMCs, PBMCs, EPCs, and CD $133^{+}$cells. We observed that all these types of cells were positive for EphB4 (data not shown). Thus, activation of EphB4 may provide an effective and innovative therapeutic strategy to improve the recruitment of injected cells to sites of neovascularization and thereby the overall efficiency of stem cell therapy.

\section{Methods}

Cell culture. EPCs were prepared from cord blood and differentiated ex vivo as previously described (15). They were grown in rat-tail type I collagencoated flasks in M199 medium (Invitrogen) supplemented with 20\% FCS (Dominique Dutscher), 25 mM HEPES (Invitrogen), antibiotic and antimycotic solution (Invitrogen), and $10 \mathrm{ng} / \mathrm{ml}$ recombinant human VEGF (R\&D Systems). All experiments with EPCs were carried out between passages 9 and 15. HUVECs were isolated from human umbilical vein by collagenase digestion and were grown in flasks coated with $0.2 \%$ gelatin in M199 medium supplemented with $15 \mathrm{mM}$ HEPES, $2 \mathrm{mM}$ glutamine, $50 \mathrm{IU} / \mathrm{ml}$ penicillin, $50 \mu \mathrm{g} / \mathrm{ml}$ streptomycin, $15 \%$ FCS, $5 \%$ human serum, $125 \mathrm{ng} / \mathrm{ml}$ amphotericin B, and $2 \mathrm{ng} / \mathrm{ml}$ bFGF (R\&D Systems). All experiments with HUVECs were carried out between passages 2 and 4 . All cultures were maintained in a humidified incubator at $37^{\circ} \mathrm{C}$ in an atmosphere containing $5 \% \mathrm{CO}_{2}$.

Table 1

EphB4 siRNA abolishes ephrin-B2-Fc-induced postischemic neovascularization

\begin{tabular}{|c|c|c|c|c|c|}
\hline & PBS & EPC + Ctrl siRNA & $\begin{array}{c}\text { EPC + Ctrl siRNA + } \\
\text { ephrin-B2-Fc }\end{array}$ & EPC + EphB4 siRNA & $\begin{array}{c}\text { EPC + EphB4 siRNA + } \\
\text { ephrin-B2-Fc }\end{array}$ \\
\hline Angiographic score & $100 \%$ & $161.5 \% \pm 10.7 \%$ & $218.8 \% \pm 12.8 \%$ & $153.5 \% \pm 2.9 \%$ & $159 \% \pm 12.5 \%{ }^{A}$ \\
\hline Cutaneous blood flow & $100 \%$ & $144.5 \% \pm 4.5 \%$ & $193.5 \% \pm 4.7 \%$ & $141.5 \% \pm 4.7 \%$ & $138.5 \% \pm 14 \%{ }^{A}$ \\
\hline Capillary density & $100 \%$ & $157.4 \% \pm 4 \%$ & $219.8 \% \pm 12.8 \%$ & $142.6 \% \pm 11.1 \%$ & $140.8 \% \pm 11 \%{ }^{A}$ \\
\hline
\end{tabular}

EPCs were transfected with siRNA and then pretreated with ephrin-B2-Fc. Six hours after induction of hind limb ischemia, these EPCs were intravenously injected into the mice $\left(1 \times 10^{6}\right.$ cells/mouse, 8 mice per group). Neovascularization was scored 12 days later as described in Methods. ${ }^{P} P<0.05$ compared with EPC plus control siRNA stimulated with ephrin-B2-Fc. Ctrl, control. 


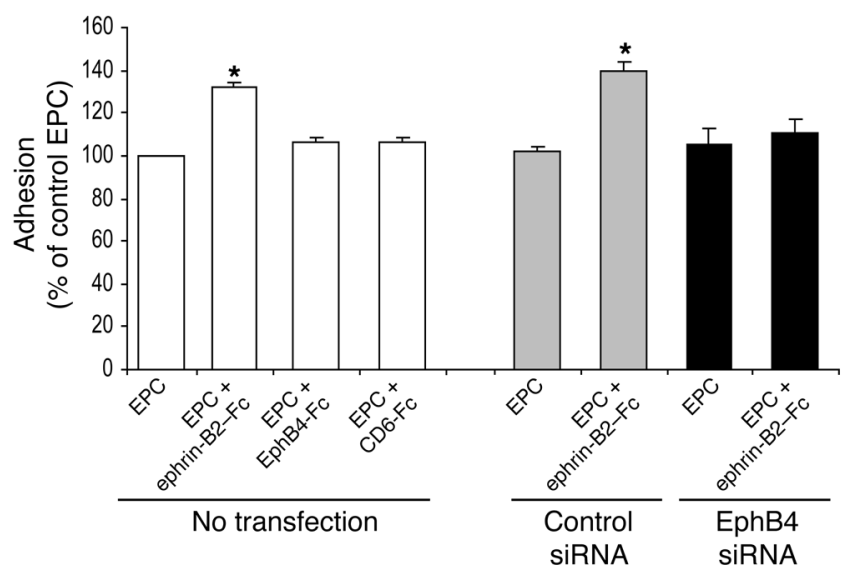

Figure 6

Ephrin-B2-Fc induces EPC adhesion to IL-1 $\beta$ prestimulated HUVEC. EPCs were stimulated for 6 hours with $3 \mu \mathrm{g} / \mathrm{ml}$ of either EphB4-Fc, ephrin-B2-Fc, or CD6-Fc or left unstimulated and then allowed to attach to IL-1 $\beta$ preactivated HUVEC monolayer. EPCs transfected with EphB4 siRNA were stimulated with $3 \mu \mathrm{g} / \mathrm{ml}$ ephrin-B2-Fc or left unstimulated and then allowed to attach in a similar manner. Cells transfected with luciferase siRNA were used as control (siRNA control cells). Data are expressed as means \pm SEM. $n=3 .{ }^{*} P<0.05$ versus nonstimulated EPCs (EPCs). EPCs represented in this figure were nonstimulated.

RNA extraction and RT-PCR. Total RNA was extracted with RNAXEL ${ }^{\mathrm{R}}$ reagent (Eurobio) according to the manufacturer's instructions. cDNA synthesis was performed with the First Strand cDNA Synthesis Kit for RT-PCR (AMV) (Boehringer Mannheim). PCR was performed with Taq polymerase (Applied Biosystems) in a PCR mix containing $1 \times$ reaction buffer, $1.5 \mathrm{mM} \mathrm{MgCl}_{2}$, $0.2 \mathrm{mM}$ deoxynucleotide mix, 0.5 units Taq polymerase, and $0.2 \mu \mathrm{M}$ forward and reverse primers. The following oligonucleotides served as primers: GAPDH forward: 5'-CCATGGAGAAGGCTGGGG-3', reverse: 5'-CAAAGTTGTCATGGATGACC-3'; EphB4 forward: 5'-CCATGGAGAAGGCTGGGG-3', reverse: 5'-CCATGTCCGATGAGATACTGTCCG-3'; ephrin-B2 forward: 5'CTGTGCCAGACCAGACCAAGA-3', reverse: $5^{\prime}$-CAGCAGAACTTGCATCTTGTC-3'; mCD62E (E selectin) forward: 5'-GCTGTCCAGTGTGAAGCCTTATC-3', reverse: 5'-GCAATGAGGATGTCAGGA-3'; mCD62P (P selectin) forward: 5'-GCTTCAGGACAATGGACAGC-3', reverse: 5'-CTTTCTTAGCAGAGCCAGGAGTG-3'; m28s forward: 5'-TTGAAAATCCGGGGGAGA$3^{\prime}$, reverse $5^{\prime}$-ACATTGTTCCAACATGCCAG-3'.

Immunocytochemistry. EPCs were grown on chamber slides (LabTech; Poly Labo) coated with rat-tail type I collagen and then fixed with $90 \%$ cold acetone solution for 10 minutes. We used the EnVision System Peroxidase/DAB kit (DAKO) to detect EphB4 and ephrin-B2 proteins. In brief, endogenous peroxidases were blocked with hydrogen peroxide, and then antibodies to EphB4 (Santa Cruz Biotechnology Inc.) or ephrin-B2 (Santa Cruz Biotechnology Inc.) were added and the chamber slides incubated for 45 minutes at room temperature. Cells were then incubated for 30 minutes at room temperature with a secondary antibody conjugated to an HRP-labeled polymer and washed with PBS. The peroxidase substrate DAB (3,3'-diaminobenzidine) was added for 10 minutes and cells counterstained with hematoxylin.

For PSGL-1 siRNA studies, EPCs were grown in 35-mm dishes until 80\% confluence and then transfected with siRNA as described below. Following transfection, cells were fixed with ice-cold acetone for 10 minutes and, after blocking with $20 \%$ normal goat serum, subsequently incubated with mouse anti-human PSGL-1 monoclonal antibody (R\&D Systems) for 1 hour at room temperature. The cells were washed with PBS and then incubated with Alexa Fluor 568-conjugated goat anti-rat IgG (Invitrogen) for 1 hour at room temperature. Nuclei were stained with DAPI. Immunostaining was visualized by fluorescence microscopy.

Mouse hind limb ischemic model. Animals were cared for in accordance with guidelines published by the NIH (Guide for the care and use of laboratory animals. NIH publication no. 85-23. Revised 1985), and the study protocol was approved by Services Vétérinaires de la Santé et de la Production Animale, Ministère de l'Agriculture, Paris, France. Seven-week-old male athymic Nude mice (Harlan) underwent surgery to induce unilateral hind limb ischemia as previously described (37). In brief, animals were anesthetized by isoflurane inhalation. The ligature was performed on the proximal origin of the right femoral artery, just above the origin of the circumflexa femoris lateralis. The mice were then randomized as follows: hind limb ischemia injected with PBS; hind limb ischemia and subsequent activated-EPC therapy; and hind limb ischemia and subsequent nonactivated-EPC therapy. Mice (8 animals per group) were then housed under specific pathogen-free conditions for 12 days. In transplantation experiments, EPCs were pretreated with $3 \mu \mathrm{g} / \mathrm{ml}$ of either ephrin-B2-Fc, EphB4-Fc, or CD6-Fc for 6 hours at $37^{\circ} \mathrm{C}$ unless otherwise stated and washed twice with PBS to remove unbound recombinant proteins. Six hours after induction of limb ischemia, EPCs were injected into the tail vein at $1 \times 10^{6}$ cells/mouse. In some experiments, EPCs were transfected with siRNA directed against EphB4 or PSGL-1 before cell stimulation and transplantation.

Laser Doppler perfusion imaging (Moor Instruments) was used to provide functional evidence for ischemia-induced changes in vascularization 12 days after injection as previously described (37). To minimize temperature variations, mice were placed on a heating platform at $37^{\circ} \mathrm{C}$. Perfusion results are expressed as a ratio of right (ischemic) to left (nonischemic) leg to control for variables including ambient light and temperature.

Vessel density was evaluated by high-definition microangiography (Trophy system). Mice were anesthetized by isoflurane inhalation. A longitudinal laparotomy was performed, and a polyethylene catheter was introduced into the abdominal aorta through which a contrast medium (barium sulfate, $1 \mathrm{~g} / \mathrm{ml}$ ) was injected. Angiography of the hind limb was then assessed, and images ( 3 per animal) were acquired by a digital $\mathrm{x}$-ray transducer. Images were then assembled to obtain a complete view of the hind limb. The vessel density is expressed as the percentage of pixels per image in the quantification area occupied by vessels. There were 3 quantification areas, defined by the external limit of the leg and placement of the ligatures on the femoral artery, the knee, and the edge of the femur.

Capillary density in the gastrocnemius muscle was assessed by immunohistochemistry as described below using rat anti-mouse CD31 antibody (BD Biosciences - Pharmingen) as the primary antibody. Sections were observed under a microscope equipped with the appropriate filters, and HistoLab software (version 5.14.1; Microvision Instruments) was used to analyze the capillary density in 5 different fields of each section.

siRNA transfection protocol. EphB4- or PSGL-1-specific siRNA duplexes (siGENOME SMARTpool) and nontargeting control siRNA (Luciferase siRNA) were purchased from Dharmacon. Transfection was performed according to the manufacturer's instructions. Briefly, EPCs were grown on 6-well plates until $80 \%$ confluence. The siRNA solution was mixed with serum-free and antibiotic-free M199 medium containing DharmaFECT2 siRNA transfection reagent. The culture medium was aspirated and replaced with $800 \mu \mathrm{l} \mathrm{fresh}$ antibiotic-free M199 medium containing 8\% FCS; $200 \mu \mathrm{l}$ of the transfection mix was added to each well to achieve a final siRNA concentration of $100 \mathrm{nM}$. Transfected cells were incubated at $37^{\circ} \mathrm{C}$ for 48 hours, and protein expression was analyzed by either Western blotting or immunofluorescence.

EPCs detection in ischemic muscle. To demonstrate homing to ischemic muscles, EPCs $\left(1 \times 10^{6}\right.$ cells/100 $\mu$ l PBS $)$ were intravenously administered 6 hours after induction of hind limb ischemia as described above. The gastrocnemius muscles were harvested 4 days after injection of EPCs. Fro- 
A
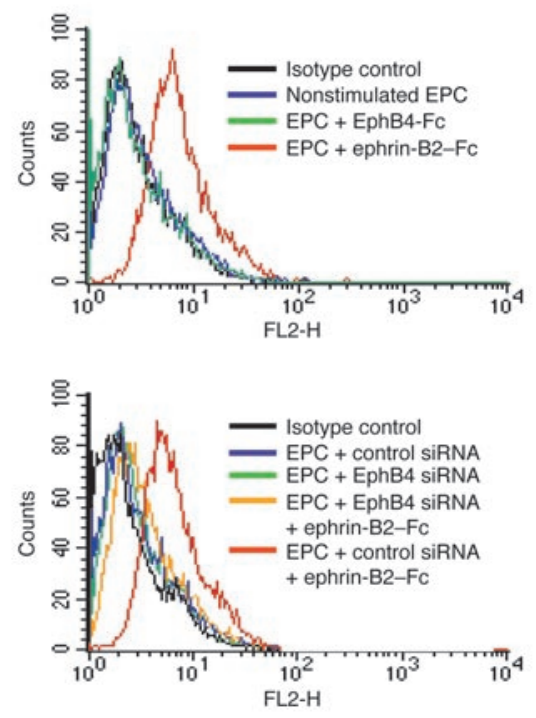

C
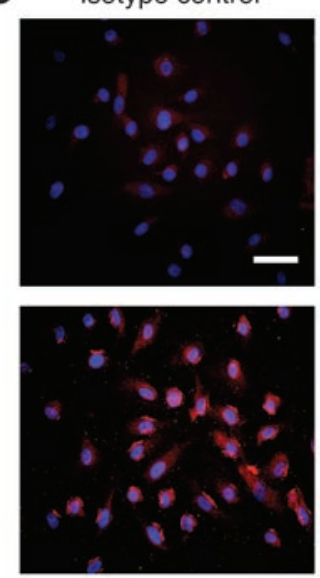

EPC + control siRNA + ephrin-B2-FC
B

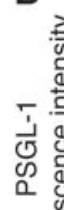

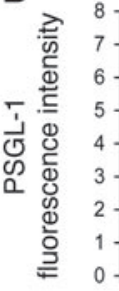

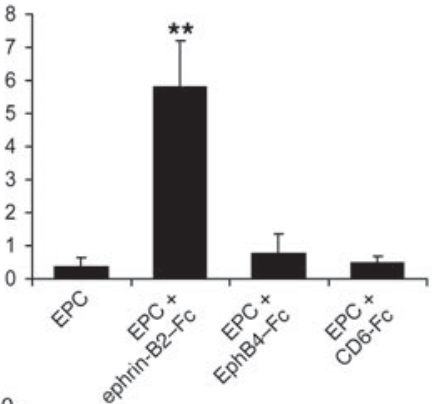

동

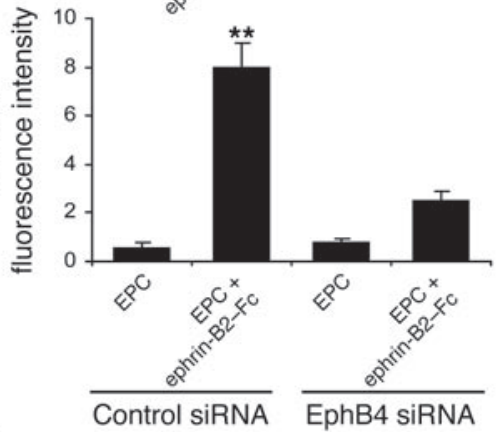

D
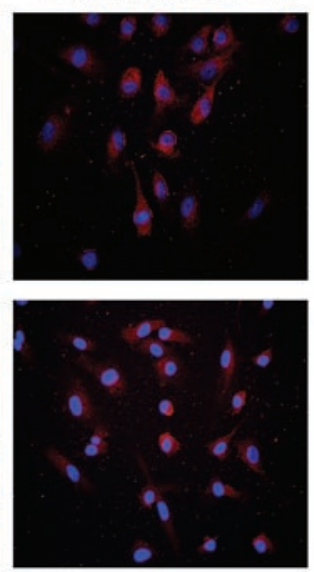

$E P C+P S G L-1$ SiRNA + ephrin-B2-Fc
Figure 7

EphB4 activation mediates EPC adhesion through PSGL-1. (A and B) PSGL-1 expression in EPCs. EPCs were stimulated with $3 \mu \mathrm{g} / \mathrm{ml}$ of either ephrin-B2Fc, EphB4-Fc, or CD6-Fc and then processed for FACS analysis of PSGL-1 expression. (A) FACS profiles of nontransfected (top panel) and EphB4 siRNA-transfected (bottom panel) EPCs. (B) Expression of PSGL-1 in nontransfected (top panel) and EphB4 siRNA-transfected (bottom panel) EPCs. $n=3$. ${ }^{* *} P<0.01$ versus nonstimulated EPCs. (C) Effect of PSGL-1 siRNA on PSGL-1 protein expression. Control siRNA- and PSGL-1 siRNA-transfected EPCs were stimulated with $3 \mu \mathrm{g} / \mathrm{ml}$ ephrin-B2-Fc or left unstimulated and then processed for immunocytochemistry with an antiPSGL-1 antibody as described in Methods. PSGL-1-positive staining appears in red. Scale bar: $20 \mu \mathrm{m}$. (D) Effect of PSGL-1 siRNA on EPC adhesion to IL-1 $\beta$ prestimulated HUVEC monolayer. Adhesion was quantified by measuring $\mathrm{OD}$ at $570 \mathrm{~nm} . n=3 .{ }^{*} P<0.05$ versus nonstimulated EPCs transfected with control siRNA. zen tissue sections $(10 \mu \mathrm{m})$ were prepared and fixed with ice-cold acetone. Incorporated EPCs were detected by immunostaining with a biotinylated anti-human CD31 antibody (Dako) followed by incubation with streptavidin-Alexa Fluor 568. To confirm incorporation of human cells, mouse vasculature was stained with anti-mouse CD31 antibody. Tissue sections were examined by laser scanning confocal microscopy (Nikon).

Immunohistochemistry. Ischemic and nonischemic gastrocnemius muscles were collected and progressively frozen in isopentane solution cooled in liquid nitrogen. Sections ( $6 \mu \mathrm{m}$ ) were fixed in 100\% cold acetone, blocked with $20 \%$ goat serum for 20 minutes, and incubated with the appropriate primary antibodies (rat anti-mouse CD31, rat anti-mouse E selectin, or rat anti-mouse P selectin) for 1 hour at room temperature. Sections were washed 3 times with PBS and incubated with goat anti-rat secondary antibodies coupled to FITC for CD31 studies or to Alexa Fluor 568 for E selectin and P selectin studies.

FACS analysis of cell-surface adhesion molecules expression. EPCs were stimulated with $3 \mu \mathrm{g} / \mathrm{ml}$ of either ephrin-B2-Fc, EphB4-Fc or CD6-Fc in M199 supplemented with $2 \% \mathrm{FCS}$ for 6 hours at $37^{\circ} \mathrm{C}$, then detached, suspended in M199 supplemented with $20 \%$ FCS, and placed on a rotating platform (50 rpm) for 3-4 hours. The EPCs were incubated for 30 minutes with FITC- or PE-labeled antibodies purchased from BD Biosciences - Pharmingen: E selectin (CD62-E), P selectin (CD62-P), PSGL-1, CD49b, CD49d, CD49e, CD51/CD61 ( $\left.\alpha_{v} \beta_{3}\right), C D 45, C D 14$, and CD18. They were then fixed in $1 \%$ paraformaldehyde, and surface expression was quantified using the FACSCalibur instrument (BD Biosciences).

Migration assay. Cell culture inserts (Millicell-PCF; Millipore) with porous polycarbonate filters (8- $\mu \mathrm{m}$ pore size) were coated with rat-tail type I collagen $(60 \mu \mathrm{g} / \mathrm{ml})$. EPCs were pretreated with $3 \mu \mathrm{g} / \mathrm{ml}$ of either ephrin-B2-Fc, EphB4-Fc, or CD6-Fc for 30 minutes at $37^{\circ} \mathrm{C}$ and washed twice with PBS. These prestimulated EPCs were suspended in M199 medium supplemented with $2 \% \mathrm{FCS}$, and $1 \times 10^{5}$ cells were added to the upper side of each insert. The inserts were placed in 12-well plates containing medium supplemented with $10 \mathrm{ng} / \mathrm{ml} \mathrm{VEGF-A}$, and cells were allowed to migrate for 4 hours at $37^{\circ} \mathrm{C}$ in an atmosphere containing $5 \% \mathrm{CO}_{2}$. The filters were then rinsed with PBS, fixed in $1 \%$ paraformaldehyde, and stained with hematoxylin. The upper surfaces of the filters were scraped with cotton swabs to remove the nonmigrating cells. The number of migrating cells attached to the lower surfaces of the filters was 
Table 2

PSGL-1 siRNA abolishes ephrin-B2-Fc-induced postischemic neovascularization

\begin{tabular}{lccccc}
\hline & PBS & EPC + Ctrl siRNA & $\begin{array}{c}\text { EPC + Ctrl siRNA + } \\
\text { ephrin-B2-Fc }\end{array}$ & EPC + PSGL-1 siRNA & $\begin{array}{c}\text { EPC + PSGL-1 siRNA + } \\
\text { ephrin-B2-Fc }\end{array}$ \\
Angiographic score & $100 \%$ & $168 \% \pm 13 \%$ & $224.1 \% \pm 23 \%$ & $156.3 \% \pm 23 \%$ & $150.3 \% \pm 13 \%{ }^{A}$ \\
Cutaneous blood flow & $100 \%$ & $153.5 \% \pm 6.7 \%$ & $214.8 \% \pm 21.3 \%$ & $149.7 \% \pm 6.1 \%$ & $154.7 \% \pm 8.7 \%{ }^{A}$ \\
Capillary density & $100 \%$ & $147.4 \% \pm 16.1 \%$ & $212.7 \% \pm 14.3 \%$ & $153.9 \% \pm 13.2 \%$ & $156.2 \% \pm 9.8 \%{ }^{A}$ \\
\hline
\end{tabular}

EPCs were transfected with siRNA and then pretreated with ephrin-B2-Fc. Six hours after induction of hind limb ischemia, these EPCs were intravenously injected into the mice $\left(1 \times 10^{6}\right.$ cells/mouse, 8 mice per group). Neovascularization was scored 12 days later as described in Methods. AP < 0.05 compared with EPCs plus control siRNA stimulated with ephrin-B2-Fc.

counted under an Axiovert 25 microscope (Zeiss). Each experiment $(n=3)$ was performed in triplicate, and 30-40 fields per filter were analyzed.

EPC adhesion on IL-1 $\beta$-activated HUVEC monolayer. Confluent HUVEC monolayer was activated with $10 \mathrm{ng} / \mathrm{ml}$ IL-1 $\beta$ (R\&D Systems) for 6 hours. A total of $3 \times 10^{4}$ cells of either nontransfected or siRNA-transfected EPCs pretreated with the fusion proteins as described in the FACS section were added to each well containing prestimulated HUVEC monolayer. After 1 hour of incubation at $37^{\circ} \mathrm{C}$, cells were washed twice with M199 medium to remove nonadhered cells, fixed in $1 \%$ glutaraldehyde, and stained with crystal violet. Adhered cells were quantified by measuring the $\mathrm{OD}$ at $570 \mathrm{~nm}$.

\section{Figure 8}

Time-dependent expression of selectins and PSGL-1. (A) Representative photomicrographs of ischemic (6 hours) and nonischemic gastrocnemius muscle sections stained with rat anti-mouse $E$ selectin or $\mathrm{P}$ selectin. Positive staining (red) is localized in blood vessels between the muscle fibers. Scale bar: $40 \mu \mathrm{m}$. (B) RT-PCR analysis of $E$ selectin and $P$ selectin mRNA expression in ischemic muscles 6 hours or 24 hours after the onset of ischemia and in nonischemic muscles at the same time intervals. Data were normalized for loading with 28S RNA. $n=3$. ${ }^{*} P<0.05 ;{ }^{* *} P<0.01$ versus nonischemic muscle. (C) PSGL-1 expression on EPCs following ephrinB2-Fc stimulation. EPCs were stimulated with ephrin-B2-Fc for 6 hours or 24 hours and then processed for FACS analysis of PSGL-1 expression. FACS profiles are shown in the left panel, and quantification of PSGL-1 expression in the right panel. $n=3$. ${ }^{*} P<0.05$ versus EPCs.
A
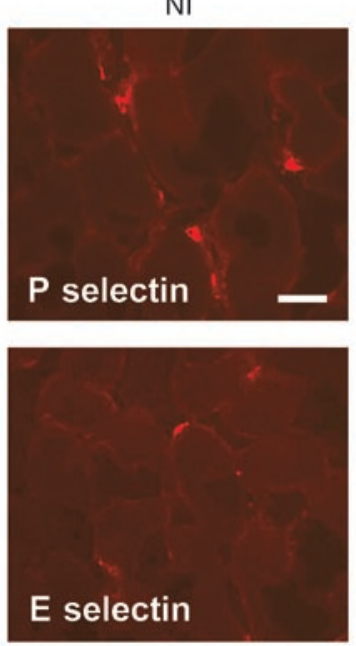

B
E selectin
$6 \mathrm{~h}$ after isch
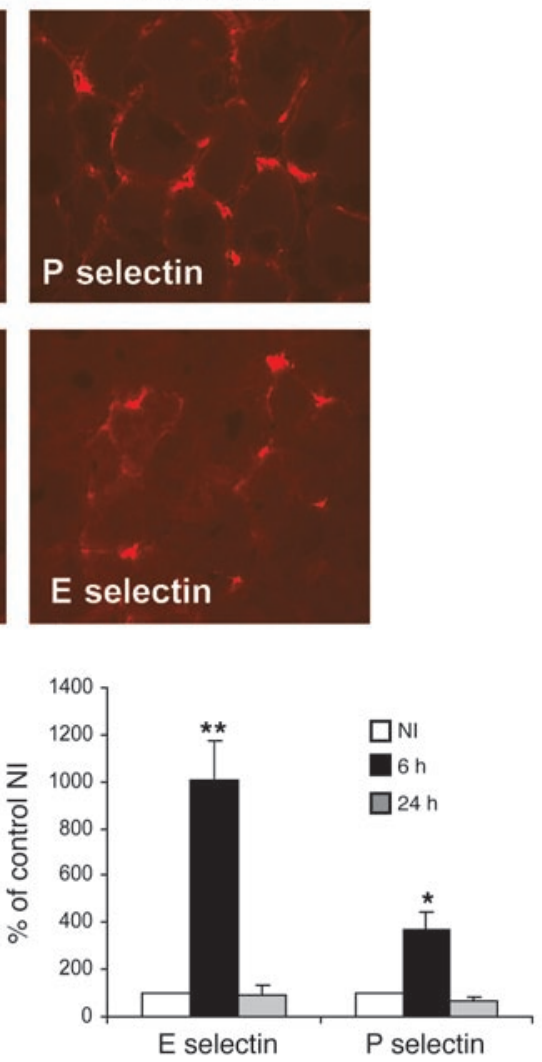

C

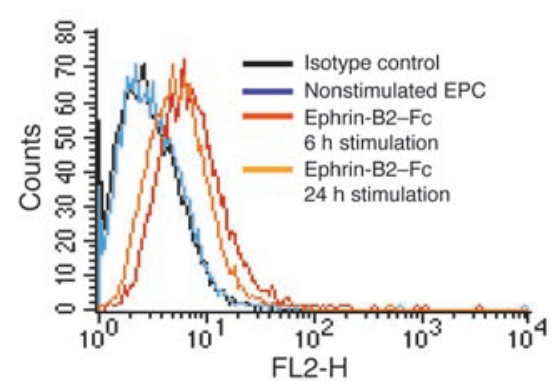

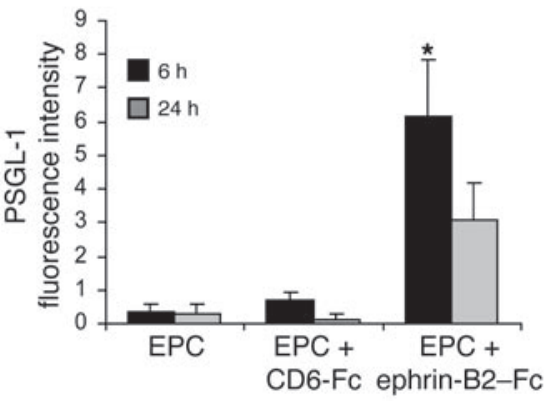




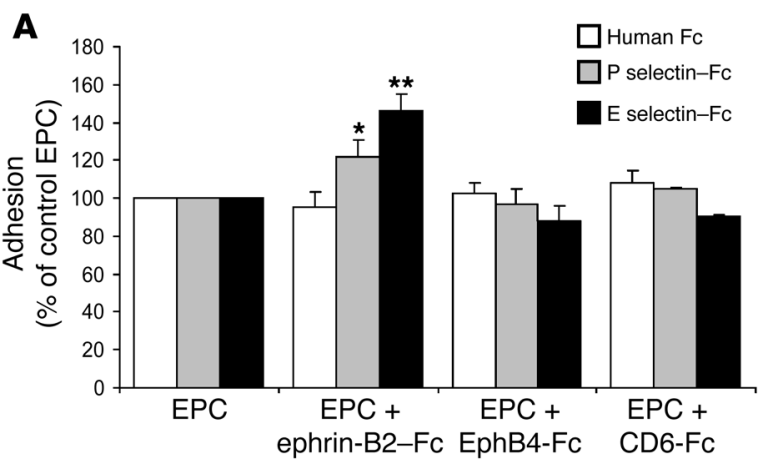

B

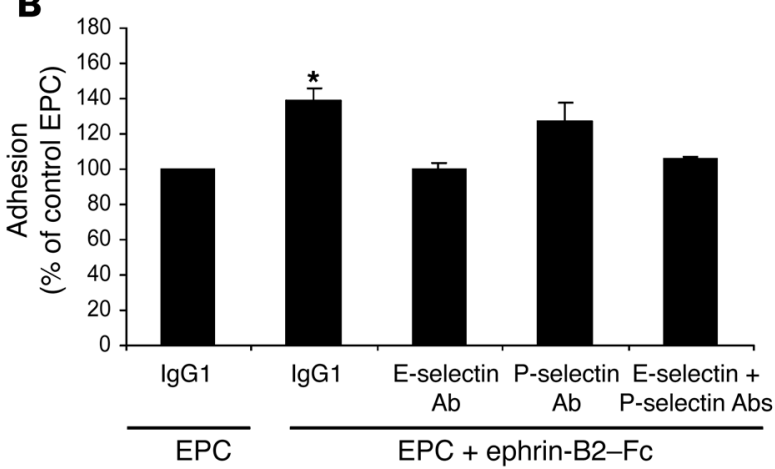

Data were expressed as the percentage of adhered cells relative to control nonstimulated EPCs. In the blocking experiments, E selectin- and P selectin-neutralizing antibodies (R\&D Systems) were added at a final concentration of $2 \mu \mathrm{g} / \mathrm{ml}$ just before EPC adhesion.

$E P C$ adhesion to recombinant $E$ selectin and $P$ selectin $-F c$ fusion proteins. The 96-well tissue culture plates were coated with $100 \mu \mathrm{l} /$ well of a $25 \mu \mathrm{g} / \mathrm{ml}$ human IgG Fc $\gamma$ solution in PBS overnight at $4{ }^{\circ} \mathrm{C}$. Unbound antibody was discarded, and wells were blocked with 1\% BSA (Sigma-Aldrich) for 1 hour at room temperature. After washing with PBS, $100 \mu \mathrm{l}$ of human recombinant E selectin or P selectin fusion proteins $(5 \mu \mathrm{g} / \mathrm{ml}$ or $10 \mu \mathrm{g} / \mathrm{ml}$ in $0.01 \%$ BSA, respectively) were added, and the plates were incubated for 1 hour at $37^{\circ} \mathrm{C}$. Then, $100 \mu \mathrm{l}$ of a $6 \times 10^{5}$ cell suspension pretreated with the fusion proteins as described for FACS analysis experiments was added and plates incubated for 1 hour at $37^{\circ} \mathrm{C}$ to allow cell adhesion. Cells were washed twice with M199 medium, fixed in $1 \%$ glutaraldehyde, and then stained with crystal violet. Cell adhesion was quantified by measuring OD at $570 \mathrm{~nm}$.

Western blotting. Cells were washed with ice-cold PBS and cell extracts pre-

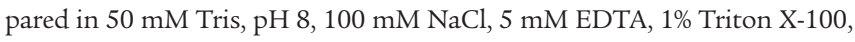
$0.5 \% \mathrm{NP}-40,1 \mathrm{mM}$ PMSF, $1 \mu \mathrm{g} / \mathrm{ml}$ leupeptin, and $1 \mu \mathrm{g} / \mathrm{ml}$ aprotinin. Protein lysates $(20 \mu \mathrm{g})$ were separated by electrophoresis in $8 \%$ acrylamide gels containing SDS and transferred to nitrocellulose membranes in $25 \mathrm{mM}$ Tris, $190 \mathrm{mM}$ glycine, and $15 \%$ methanol. Membranes were blocked by incubation in $25 \mathrm{mM}$ Tris-buffered saline containing $0.1 \%$ Tween-20 (TBST) and 5\% non-

\section{Figure 10}

Schematic model showing how EphB4 activation increases adhesion potential of EPCs. E selectin and P selectin were overexpressed by the ischemic endothelium. EphB4 activation enhances PSGL-1 expression at the surface of EPCs. This allows attachment of the circulating ephrin-B2-Fc-stimulated EPCs via interaction between PSGL-1 and $E$ selectin and $P$ selectin. The attached cells can then migrate to the ischemic tissue where they can integrate into the nascent vessels and/or participate in a paracrine fashion in the neoangiogenic process.

\section{Figure 9}

E selectin and $P$ selectin mediate ephrin-B2-FC-induced EPC adhesion. (A) Ephrin-B2-Fc treatment increased EPC adhesion to immobilized E selectin and $P$ selectin fusion proteins. EPCs were stimulated with $3 \mu \mathrm{g} / \mathrm{ml}$ of either ephrin-B2-Fc, EphB4-Fc, or CD6-Fc. Then the cells were allowed to adhere to immobilized recombinant E selectin-Fc (black bars), P selectin-Fc (gray bars), or to human IgG Fc $\gamma$ fragments (white bars). Adhesion was quantified by measuring OD at $570 \mathrm{~nm}$. Results are expressed as percentages of control nonstimulated EPCs (EPC). EPCs represented in this figure were nonstimulated. $n=3$. ${ }^{*} P<0.05$; ${ }^{\star \star} P<0.01$ versus nonstimulated EPCs. (B) Blocking antibodies directed against $E$ selectin and $P$ selectin neutralized ephrin-B2-Fc-induced EPC adhesion to IL-1 $\beta$-prestimulated HUVEC monolayers. $n=3$. ${ }^{\star} P<0.05$ versus EPCs treated with control IgG1.

fat milk powder for 2 hours at room temperature. They were then incubated for 1 hour at room temperature with the appropriate primary antibodies in TBST containing 5\% nonfat milk powder. The membranes were washed 3 times in TBST and then incubated with HRP-conjugated anti-rabbit IgG in TBST for 1 hour at room temperature. The signal was revealed with the Pierce Enhanced Chemiluminescence System (ECL), used as recommended by the manufacturer. Quantification was done with MultiGauge software (version 2.3; Fujifilm).

Immunoprecipitation and tyrosine phosphorylation assay. For tyrosine phosphorylation assay, EPCs grown to $80 \%$ confluence in $15-\mathrm{cm}$ dishes were starved with M199 medium containing 2\% FCS for 16 hours and then with serum-free M199 medium for 2 hours. After starving, the cells were stimulated with $3 \mu \mathrm{g} / \mathrm{ml}$ ephrin-B2-Fc in serum-free M199 medium for 30 minutes to activate the EphB4 receptor. The medium was aspirated and cells were washed twice with cold PBS. Cell lysates were then immediately prepared in protein extraction buffer consisting of $50 \mathrm{mM}$ Tris- $\mathrm{HCl}, \mathrm{pH} 7.4,150 \mathrm{mM}$ $\mathrm{NaCl}, 1 \mathrm{mM}$ EDTA, 0.25\% deoxycholic acid, 1\% NP-40, $10 \mathrm{mM} \mathrm{NaF}, 2 \mathrm{mM}$ sodium vanadate, $20 \mathrm{mM}$ glycerophosphate, and a protease inhibitor cocktail. Protein extracts were clarified by centrifugation at $12,000 \mathrm{~g}$ for $15 \mathrm{~min}$ utes at $4^{\circ} \mathrm{C}$, and clarified protein samples were incubated for 2 hours at $4{ }^{\circ} \mathrm{C}$ with protein A-sepharose precoated with an anti-EphB4 antibody (Zymed Laboratories). The precipitated immune complexes were washed 3 times with the same extraction buffer, solubilized in $1 \times$ SDS-PAGE loading buffer by boiling, separated on $10 \%$ SDS-PAGE, and transferred to nitrocellulose membranes in $25 \mathrm{mM}$ Tris, $190 \mathrm{mM}$ glycine, and 15\% methanol. Membranes

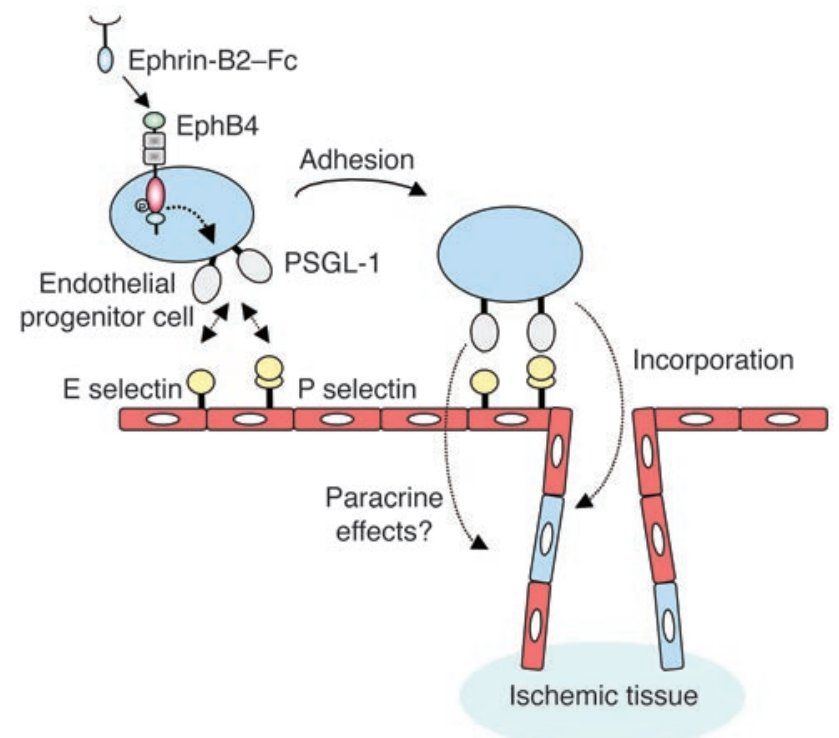


were then processed for Western blotting with an anti-phosphotyrosine antibody (4G10; Upstate USA Inc.) as described above. To check for equal protein loading, membranes were probed with an EphB4 antibody (R\&D Systems).

Immunofluorescence localization of tyrosine-phosphorylated EphB4. EPCs grown to $80 \%$ confluence in $35-\mathrm{mm}$ dishes were serum starved and then stimulated with $3 \mu \mathrm{g} / \mathrm{ml}$ ephrin-B2-Fc as described for the tyrosine phosphorylation assay (see above). Media were then aspirated and cells were washed twice with cold PBS, fixed in $4 \%$ paraformaldehyde for 10 minutes, permeabilized with $0.2 \%$ Triton X-100, and blocked with $20 \%$ FCS. To visualize EphB4, cells were incubated with $3 \mu \mathrm{g} / \mathrm{ml}$ ephrin-B2-Fc for 1 hour at room temperature and then with a goat anti-human $\mathrm{Fc}$ fragment antibody (Jackson ImmunoResearch Laboratories Inc.). Binding was revealed with an anti-goat antibody conjugated to Alexa Fluor 488. Incubation with an anti-phosphotyrosine antibody (4G10; Upstate USA Inc.) followed by an anti-mouse antibody conjugated to Alexa Fluor 555 was used to stain for phosphotyrosine proteins.

Statistics. Results are expressed as mean \pm SEM. One-way ANOVA was used to compare variables. A value of $P<0.05$ was considered significant.

\section{Acknowledgments}

We thank Dong You and Ludovic Waeckel for their technical help. We also thank Patrice Castagnet (Laboratoire
d'Anatomopathologie at Lariboisière Hospital, Paris, France) for his excellent assistance in tissue embedding and processing. We are grateful to the maternity hospital of Lariboisière Hospital for providing us with cord blood samples. This work was supported in part by grants from the Agence Nationale de la Recherche (Cardiovascular, Obesity, and Diabetes; ANR-05028-01 and ANR-05-022-01) and the Del Duca Foundation. J.S. Silvestre was supported by grants from the Agence Nationale de la Recherche (Cardiovascular, Obesity, and Diabetes; ANR05-JCJC-0065-01).

Received for publication February 24, 2006, and accepted in revised form February 28, 2007.

Address correspondence to: Gérard Tobelem, Institut des Vaisseaux et du Sang, 8 rue Guy Patin, 75475 Paris Cedex 10, France. Phone: 33-1-45-26-21-98; Fax: 33-1-42-82-94-73; E-mail: gerard. tobelem@lrb.aphp.fr.

Philippe Foubert and Jean-Sébastien Silvestre contributed equally to this work.
1. Kalka, C., et al. 2000. Transplantation of ex vivo expanded endothelial progenitor cells for therapeutic neovascularization. Proc. Natl. Acad. Sci. U. S. A. 97:3422-3427.

2. Kawamoto, A., et al. 2001. Therapeutic potential of ex vivo expanded endothelial progenitor cells for myocardial ischemia. Circulation. 103:634-637.

3. Asahara, T., et al. 1999. Bone marrow origin of endothelial progenitor cells responsible for postnatal vasculogenesis in physiological and pathological neovascularization. Circ. Res. 85:221-228.

4. Murohara, T., et al. 2000. Transplanted cord blood-derived endothelial precursor cells augment postnatal neovascularization. J. Clin. Invest. 105:1527-1536.

5. Hristov, M., and Weber, C. 2004. Endothelial progenitor cells: characterization, pathophysiology, and possible clinical relevance. J. Cell. Mol. Med. 8:498-508.

6. Aicher, A., et al. 2003. Assessment of the tissue distribution of transplanted human endothelial progenitor cells by radioactive labeling. Circulation. 107:2134-2139.

7. Kureishi, Y., et al. 2000. The HMG-CoA reductase inhibitor simvastatin activates the protein kinase Akt and promotes angiogenesis in normocholesterolemic animals. Nat. Med. 6:1004-1010.

8. Napoli, C., et al. 2005. Beneficial effects of concurrent autologous bone marrow cell therapy and metabolic intervention in ischemia-induced angiogenesis in the mouse hindlimb. Proc. Natl. Acad. Sci. U. S. A. 102:17202-17206.

9. Akita, T., et al. 2003. Hypoxic preconditioning augments efficacy of human endothelial progenitor cells for therapeutic neovascularization. Lab. Invest. 83:65-73.

10. Dodelet, V.C., and Pasquale, E.B. 2000. Eph receptors and ephrin ligands: embryogenesis to tumorigenesis. Oncogene. 19:5614-5619.

11. Wang, H.U., Chen, Z.F., and Anderson, D.J. 1998. Molecular distinction and angiogenic interaction between embryonic arteries and veins revealed by ephrin-B2 and its receptor Eph-B4. Cell. 93:741-753.

12. Adams, R.H., et al. 1999. Roles of ephrinB ligands and EphB receptors in cardiovascular development: demarcation of arterial/venous domains, vascular morphogenesis, and sprouting angiogenesis. Genes Dev. 13:295-306.

13. Gerety, S.S., Wang, H.U., Chen, Z.F., and Anderson,
D.J. 1999. Symmetrical mutant phenotypes of the receptor EphB4 and its specific transmembrane ligand ephrin-B2 in cardiovascular development. Mol. Cell. 4:403-414.

14. Davy, A., and Soriano, P. 2005. Ephrin signaling in vivo: look both ways. Dev. Dyn. 232:1-10.

15. Ricousse-Roussanne, S., et al. 2004. Ex vivo differentiated endothelial and smooth muscle cells from human cord blood progenitors home to the angiogenic tumor vasculature. Cardiovasc. Res. 62:176-184.

16. Zimmer, M., Palmer, A., Kohler, J., and Klein, R. 2003. EphB-ephrinB bi-directional endocytosis terminates adhesion allowing contact mediated repulsion. Nat. Cell Biol. 5:869-878.

17. Urbich, C., and Dimmeler, S. 2004. Endothelial progenitor cells: characterization and role in vascular biology. Circ. Res. 95:343-353.

18. Kamihata, H., et al. 2001. Implantation of bone marrow mononuclear cells into ischemic myocardium enhances collateral perfusion and regional function via side supply of angioblasts, angiogenic ligands, and cytokines. Circulation. 104:1046-1052.

19. Kinnaird, T., et al. 2004. Local delivery of marrowderived stromal cells augments collateral perfusion through paracrine mechanisms. Circulation. 109:1543-1549.

20. Kansas, G.S. 1996. Selectins and their ligands: current concepts and controversies. Blood. 88:3259-3287.

21. Yang, C., et al. 2004. Enhancement of neovascularization with cord blood CD133+ cell-derived endothelial progenitor cell transplantation. Thromb. Haemost. 91:1202-1212.

22. Egami, K., Murohara, T., Aoki, M., and Matsuishi, T. 2006. Ischemia-induced angiogenesis: role of inflammatory response mediated by P-selectin. J. Lenkoc. Biol. 79:971-976.

23. Hayashi, S., Asahara, T., Masuda, H., Isner, J.M., and Losordo, D.W. 2005. Functional ephrin-B2 expression for promotive interaction between arterial and venous vessels in postnatal neovascularization. Circulation. 111:2210-2218.

24. Pasquale, E.B. 2004. Eph-ephrin promiscuity is now crystal clear. Nat. Neurosci. 7:417-418.

25. Urbich, C., et al. 2005. Soluble factors released by endothelial progenitor cells promote migration of endothelial cells and cardiac resident progenitor cells. J. Mol. Cell. Cardiol. 39:733-742.
26. Chavakis, E., et al. 2005. Role of beta2-integrins for homing and neovascularization capacity of endothelial progenitor cells. J. Exp. Med. 201:63-72.

27. Walter, D.H., et al. 2002. Statin therapy accelerates reendothelialization: a novel effect involving mobilization and incorporation of bone marrowderived endothelial progenitor cells. Circulation. 105:3017-3024.

28. Vajkoczy, P., et al. 2003. Multistep nature of microvascular recruitment of ex vivo-expanded embryonic endothelial progenitor cells during tumor angiogenesis. J. Exp. Med. 197:1755-1765.

29. Hidalgo, A., Weiss, L.A., and Frenette, P.S. 2002. Functional selectin ligands mediating human $\mathrm{CD} 34^{+}$cell interactions with bone marrow endothelium are enhanced postnatally. J. Clin. Invest. 110:559-569. doi:10.1172/JCI200214047.

30. Rossi, F.M., et al. 2005. Recruitment of adult thymic progenitors is regulated by P-selectin and its ligand PSGL-1. Nat. Immunol. 6:626-634.

31. Steinle, J.J., et al. 2002. Eph B4 receptor signaling mediates endothelial cell migration and proliferation via the phosphatidylinositol 3-kinase pathway. J. Biol. Chem. 277:43830-43835.

32. McEver, R.P., and Cummings, R.D. 1997. Perspectives series: cell adhesion in vascular biology. Role of PSGL-1 binding to selectins in leukocyte recruitment. J. Clin. Invest. 100:485-491.

33. Heiss, C., et al. 2005. Impaired progenitor cell activity in age-related endothelial dysfunction. J. Am. Coll. Cardiol. 45:1441-1448.

34. Vasa, M., et al. 2001. Number and migratory activity of circulating endothelial progenitor cells inversely correlate with risk factors for coronary artery disease. Circ. Res. 89:E1-E7.

35. Tepper, O.M., et al. 2002. Human endothelial progenitor cells from type II diabetics exhibit impaired proliferation, adhesion, and incorporation into vascular structures. Circulation. 106:2781-2786.

36. Tamarat, R., et al. 2004. Impairment in ischemiainduced neovascularization in diabetes: bone marrow mononuclear cell dysfunction and therapeutic potential of placenta growth factor treatment. Am. J. Pathol. 164:457-466.

37. Silvestre, J.S., et al. 2001. Proangiogenic effect of angiotensin-converting enzyme inhibition is mediated by the bradykinin $\mathrm{B}(2)$ receptor pathway. Circ. Res. 89:678-683. 